\title{
Comparison of lymphoblast mitochondria from normal subjects and patients with Barth syndrome using electron microscopic tomography
}

\author{
Devrim Acehan ${ }^{1}$, Yang $\mathrm{Xu}^{2}$, David L Stokes ${ }^{1,3}$ and Michael Schlame ${ }^{1,2}$ \\ ${ }^{1}$ Skirball Institute and Department of Cell Biology, New York University School of Medicine, New York, \\ NY, USA; ${ }^{2}$ Department of Anesthesiology, New York University School of Medicine, New York, NY, USA and \\ ${ }^{3}$ New York Structural Biology Center, New York, NY, USA
}

\begin{abstract}
Barth syndrome (BTHS) is a mitochondrial disorder that is caused by mutations in the tafazzin gene, which affects phospholipid composition. To determine whether this defect leads to alterations in the internal threedimensional organization of mitochondrial membranes, we applied electron microscopic tomography to lymphoblast mitochondria from BTHS patients and controls. Tomograms were formed from 50 and $150 \mathrm{~nm}$ sections of chemically fixed lymphoblasts and the data were used to manually segment volumes of relevant structural details. Normal lymphoblast mitochondria contained well-aligned, lamellar cristae with slot-like junctions to the inner boundary membrane. In BTHS, mitochondrial size was more variable and the total mitochondrial volume per cell increased mainly due to clusters of fragmented mitochondria inside nuclear invaginations. However, mitochondria showed reduced cristae density, less cristae alignment, and inhomogeneous cristae distribution. Three-dimensional reconstruction of BTHS mitochondria revealed zones of adhesion of the opposing inner membranes, causing obliteration of the intracrista space. We found small isolated patches of adhesion as well as extended adhesion zones, resulting in sheets of collapsed cristae packaged in multiple concentric layers. We also found large tubular structures (diameter 30-150 nm) that appeared to be derivatives of the adhesion zones. The data suggest that mitochondrial abnormalities of BTHS involve adhesions of inner mitochondrial membranes with subsequent collapse of the intracristae space. Laboratory Investigation (2007) 87, 40-48. doi:10.1038/labinvest.3700480; published online 16 October 2006
\end{abstract}

Keywords: cardiomyopathy; electron microscopic tomography; mitochondrial disease; mitochondrial ultrastructure; skeletal myopathy

Barth syndrome (BTHS) is a mitochondrial disorder presenting with cardiomyopathy, skeletal muscle weakness, neutropenia, and growth retardation. ${ }^{1-3}$ This syndrome is caused by mutations in the tafazzin gene that has an X-linked recessive inheritance pattern. ${ }^{4}$ Although tafazzin is lacking a typical mitochondrial targeting signal, its mitochondrial localization has been demonstrated in yeast. ${ }^{5-7}$ The amino-acid sequence of tafazzin contains an acyltransferase motif, suggesting its involvement in lipid metabolism. ${ }^{8}$ Indeed, altered phospholipid composition was found in heart, skeletal muscle, platelets,

Correspondence: Dr M Schlame, MD, Department of Anesthesiology, New York University School of Medicine, 550 First Avenue, New York, NY 10016, USA.

E-mail: michael.schlame@med.nyu.edu

Received 28 May 2006; revised 22 August 2006; accepted 27 August 2006; published online 16 October 2006 lymphoblasts, and fibroblasts of BTHS patients. ${ }^{9-11}$ The phospholipid most affected by the disease is cardiolipin.

Cardiolipin is a phospholipid dimer with four fatty acids that is specifically localized in mitochondria. Cardiolipin is tightly bound to virtually all major proteins of the inner mitochondrial membrane, notably the solute carriers, the $\mathrm{F}_{0} \mathrm{~F}_{1}$-ATPase, as well as respiratory complex III and IV (for a review, see Schlame et al). ${ }^{12}$ Only in the presence of cardiolipin are the respiratory complexes able to form stable oligomeric supercomplexes. ${ }^{13,14}$ Thus, cardiolipin is thought to play an essential role in the supramolecular organization of the inner mitochondrial membrane. In most cardiolipin molecular species, only one or two types of fatty acids exist, which results in a high degree of molecular symmetry and structural uniformity. ${ }^{15}$ Cardiolipin molecules from patients with BTHS, however, lack 
these features; instead, they form multiple molecular species due to random attachment of fatty acids. ${ }^{15}$ BTHS patients also have a lower total concentration of cardiolipin. For instance, in lymphoblasts, cardiolipin decreases from 5.4 to $2.8 \%$ of total phospholipids. ${ }^{11}$

Given the role cardiolipin plays in the structural organization of the inner mitochondrial membrane, BTHS ought to have consequences for mitochondrial function and morphology. Indeed, tissue biopsies from BTHS patients have revealed mitochondria with pathologic cristae. ${ }^{1,16-19}$ Furthermore, a decrease in coupling efficiency between respiration and phosphorylation has been found. ${ }^{5,20}$ In this study, we compare normal and BTHS lymphoblast mitochondria using electron microscopic tomography. This technique reveals aspects of the threedimensional (3-D) mitochondrial structure, which remain unrecognized in conventional electron microscopy. ${ }^{21-24}$

\section{Materials and methods}

\section{Patients}

Collection of blood samples was approved by institutional review boards of Johns Hopkins University and New York University Medical Center. The study was performed with four BTHS patients and four control subjects (age 1-9 years). Owing to the X-linked recessive nature of BTHS, all subjects were male. BTHS patients had a mutation in the tafazzin gene, which caused deletion of the protein. Patients presented with cardiomyopathy, and at least two of the noncardiac symptoms of BTHS, including skeletal myopathy, neutropenia, and growth retardation. Control subjects had a normal tafazzin gene and none of the symptoms of BTHS. They typically presented for work-up of unrelated neurologic conditions.

\section{Lymphoblast Cultures}

Lymphoblast cell lines were established by EpsteinBarr virus transformation of leukocytes isolated from whole blood using Ficoll-Hypaque gradients. The cell lines were cultured in RPMI 1640 medium at $37^{\circ} \mathrm{C}$, in the presence of $10 \%$ fetal bovine serum, $50 \mathrm{IU} / \mathrm{ml}$ penicillin, $0.05 \mathrm{mg} / \mathrm{ml}$ streptomycin, and $5 \% \mathrm{CO}_{2}$. The serum was heat-inactivated at $56^{\circ} \mathrm{C}$ for 30 min prior to use. Lymphoblasts were seeded at a density of $3 \times 10^{5}$ cells $/ \mathrm{ml}$ and cultures were expanded every $2-3$ days.

\section{Specimen Preparation}

Lymphoblasts were pelleted using a bench-top centrifuge operated at 3000 r.p.m. The pellets were washed with $0.1 \mathrm{M}$ sodium cacodylate buffer $(\mathrm{pH}$ $7.4)$ and fixed for $30 \mathrm{~min}$ in primary fixative $(3 \%$ glutaraldehyde, $0.2 \%$ tannic acid in $0.1 \mathrm{M}$ sodium cacodylate buffer, $\mathrm{pH}$ 7.4) at room temperature. Pellets were washed three times for $10 \mathrm{~min}$ in $0.1 \mathrm{M}$ sodium cacodylate buffer and then incubated for $60 \mathrm{~min}$ on ice in the secondary fixative $\left(1 \% \mathrm{OsO}_{4}\right.$ in $0.1 \mathrm{M}$ sodium cacodylate buffer, $\mathrm{pH}$ 7.4). The cell pellets were washed again three times for $10 \mathrm{~min}$ in $0.1 \mathrm{M}$ sodium cacodylate buffer and then rinsed with water. Pellets were further fixed and stained en bloc in $1 \%$ aqueous uranyl acetate for $60 \mathrm{~min}$. After the samples were fully dehydrated with a graded ethanol series (from 10 to $100 \%$ ) at progressively lower temperatures (from $20^{\circ} \mathrm{C}$ to $-35^{\circ} \mathrm{C}$ ), they were infiltrated with LX-12 resin over a period of $18 \mathrm{~h}$, slowly increasing the resin content to $100 \%$. Resin blocks were heat cured at $60^{\circ} \mathrm{C}$ for $48 \mathrm{~h}$ and then sectioned to a thickness of either 50 or $150 \mathrm{~nm}$. Sections were collected on plastic-coated copper EM grids and stained for $10 \mathrm{~min}$ with $2 \%$ uranyl acetate in water and for $2 \mathrm{~min}$ with Sato Lead Solution. Colloidal gold $(10 \mathrm{~nm})$ was randomly spread across the sections after treatment with $0.02 \%$ poly-L-lysine for $10 \mathrm{~min}$. The sections were washed with water, and finally coated with a thin layer of evaporated carbon. Cell lines from controls and BTHS patients were prepared in parallel using the same conditions at every step.

\section{Electron Microscopic Tomography}

Grids were mounted on a high tilt sample holder (Fischione, Export, PA, USA) and the samples were imaged at 13000-16000 nominal magnification, using a Tecnai TF20 electron microscope (FEI Corporation, Hillsboro, OR, USA) equipped with a $4 \mathrm{k} \times 4 \mathrm{k}$ CCD camera (TVIPS, Gauting, Germany). Tilt series were collected from -70 to 70 degrees, in oneor two-degree increments along two perpendicular tilt axes at 1.0-1.5 $\mu \mathrm{m}$ defocus, using EMMENU (TVIPS). Images from the tilt series were used to calculate 3-D density maps using the IMOD tomography package. ${ }^{25}$ Colloidal gold particles were used for fiducial alignment of images within each tilt series. Independent tomograms were calculated with data from each of the two orthogonal tilt axes, which were then combined according to the standard IMOD practice. Final combined tomograms were viewed using the program AMIRA (Mercury Computer Systems, San Diego, CA, USA). Features of interest were manually labeled on tomogram slices using the segmentation module to create colored 3-D renderings.

\section{Results}

First we examined mitochondria in normal lymphoblasts. As reported previously, ${ }^{20}$ mitochondria were localized in two subcellular compartments: near the plasma membrane and inside the nuclear invagination that is typically present in lymphoblasts 
(Figure 1a). The size of mitochondrial cross-sections varied between 0.1 and $2 \mu \mathrm{m}^{2}$ (average: $0.48 \mu \mathrm{m}^{2}$ ). Mitochondria contained groups of parallel cristae, which often extended through the entire body of the organelle (Figure 1b and c). We constructed tomograms of $50 \mathrm{~nm}$-thick sections through mitochondria for 3-D structural analysis. These tomograms showed that the cristae had a predominantly lamellar architecture (Figure 2a-c). Cristae connected to the inner boundary membrane via lamellar junctions. These junctions were slot-like segments that protruded from the main crista body (Figure $2 \mathrm{~b}$ and c). In addition, lymphoblast mitochondria contained tubular elements, which appeared to be outgrowths of lamellar cristae (Figure 2c and d). Some of these tubules were at least $50 \mathrm{~nm}$ long, as they penetrated the entire depth of the reconstructed volume.

In lymphoblasts from BTHS patients, the total mitochondrial volume expanded, particularly inside the nuclear invagination (Figure 1d). This observation was consistent with our previously published data, which we interpreted as mitochondrial proliferation, ${ }^{20}$ but mitochondrial swelling (Figure $1 \mathrm{~d}-\mathrm{f}$ ) also may have contributed to the expansion of the mitochondrial volume. Furthermore, the size of mitochondrial cross-sections was more heterogeneous (Figure 1e and f). For instance, we found that the proportion of lymphoblasts that contained 20 or more mitochondrial cross-sections increased from $6 \%$ in controls to $38 \%$ in BTHS patients. At the same time, the number of giant mitochondria (crosssections larger than $3 \mu \mathrm{m}^{2}$ ) rose from 11 per 100 cellular cross-sections in controls to 48 per 100 cellular cross-sections in BTHS.

A typical cluster of pathologic BTHS mitochondria is shown in Figure 3. These mitochondria have a distinct morphology with a striking lack of inner membranes and a very poor alignment of cristae. The remaining cristae were concentrated around the mitochondrial periphery (Figure 3a). 3-D reconstructions of these mitochondria showed that the cristae retained a lamellar shape, but they were much more interconnected than in normal mitochondria, and they contained segments that were swollen or collapsed. In the collapsed segments, the intracrista space was completely obliterated due to close adhesion of the opposing inner membranes (Figure 3b-d). Furthermore, the central cavity of mitochondria contained a distinct population of membranes
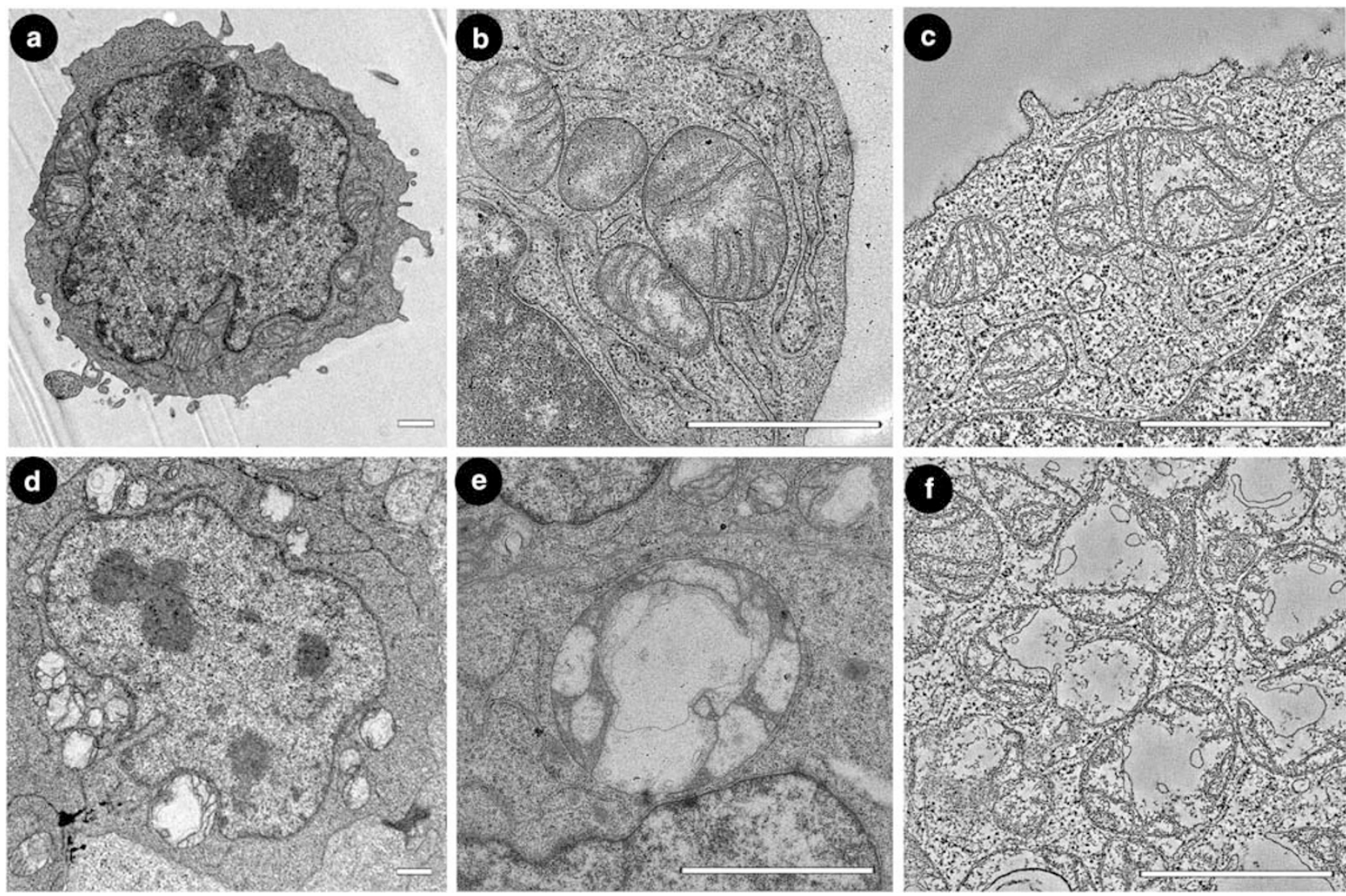

Figure 1 Mitochondria in lymphoblasts from normal individuals (a-c) and BTHS patients (d-f). (a) Electron micrograph of a normal lymphoblast. (b) Electron micrograph of a cluster of normal mitochondria. (c) Slice of a tomogram showing several normal mitochondria. (d) Electron micrograph of a BTHS lymphoblast with degenerated mitochondria of variable size. (e) Electron micrograph showing a giant mitochondrion inside the nuclear invagination of a BTHS lymphoblast. (f) Slice of a tomogram showing fragmented mitochondria of a BTHS lymphoblast. Bars: $1 \mu \mathrm{m}$. 


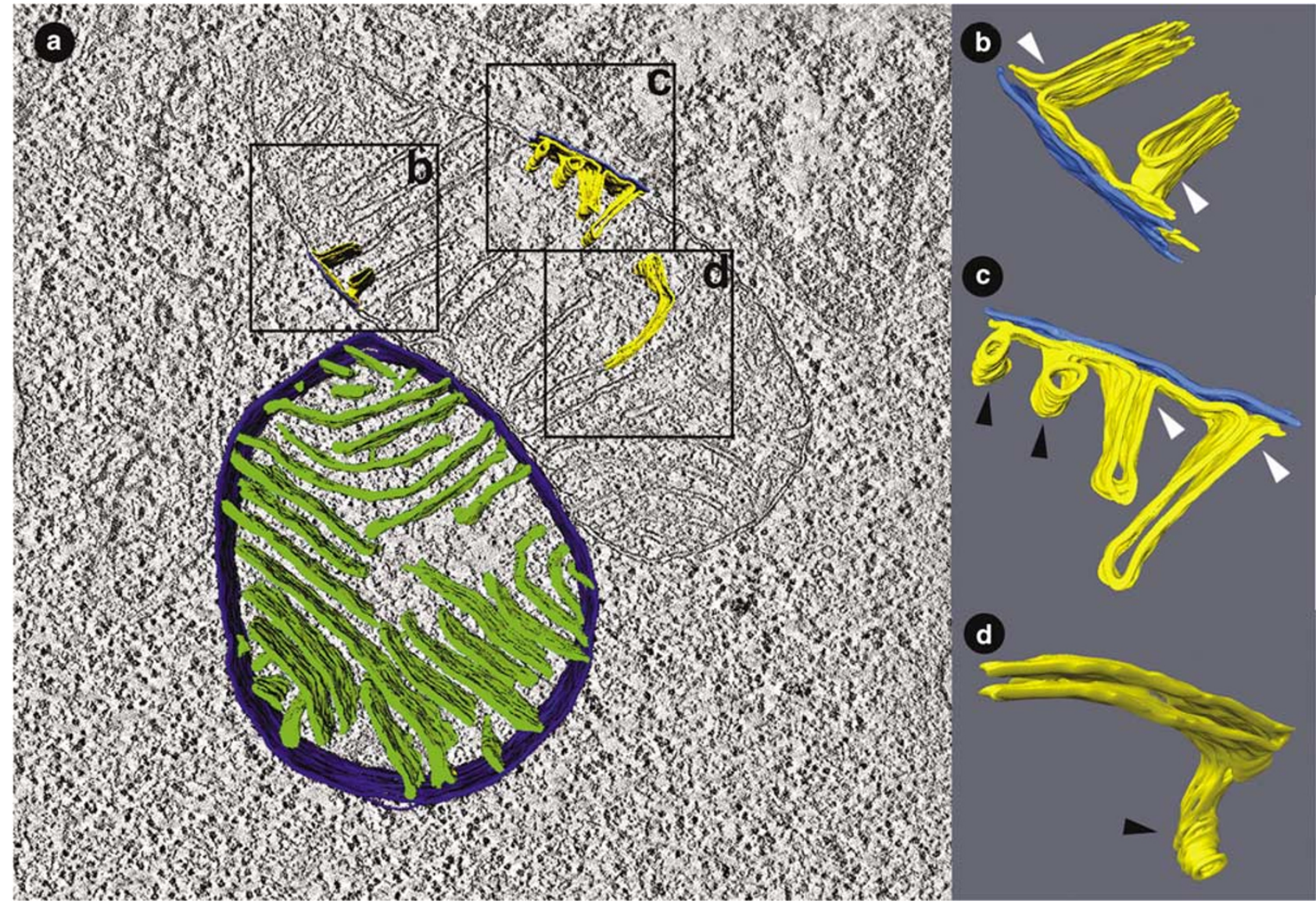

Figure 2 Tomogram of two normal lymphoblast mitochondria ( $50 \mathrm{~nm}$ section). (a) Slice of the tomogram with surface-rendered depiction of a 3-D model, showing the peripheral compartment (outer membrane + intermembrane space + inner boundary membrane) in dark blue and the cristae in green. The boxes $(0.4 \times 0.4 \mu \mathrm{m})$ mark three segmented details, in which the outer membrane is shown in bright blue and the inner membrane in yellow. (b, c, d) 3-D models of membranes in the corresponding boxes. The images were magnified and rotated to reveal details of interest. White arrowheads point to junctions between lamellar cristae and the inner boundary membrane. Black arrowheads point to tubular cristae.

that formed wide-open tubes with a diameter of 30-150 nm (orange structures in Figure 3). These membranes appeared to be connected to the collapsed cristae (Figure 3b-d).

As mentioned above, giant mitochondria were observed more frequently in BTHS lymphoblasts. Some giant mitochondria had an onion-shaped morphology, in which we identified two zones with distinct cristae architecture. The first zone contained lamellar cristae that wrapped around the core of the organelle in multiple layers. The second zone contained a web of interconnected cristae with honeycomb appearance, in which reversal of the inner membrane curvature forced the matrix into tubular shape (Figure 4a). Both zones typically coexisted in a single giant mitochondrion. Giant mitochondria also displayed evidence of inner membrane adhesion. We found small patches of crista membrane contact zones (Figure $4 \mathrm{~b}$ and c) as well as extended regions of membrane adhesion (Figure 4d). Morphological details of collapsed cristae are depicted in a collage of tomogram slices in Figure 5. The slices show that normal crista segments alternate with collapsed crista segments and that the collapse results from close apposition of the two membranes.

We also constructed tomograms of $150 \mathrm{~nm}$-thick sections in order to probe the structural depth of cristae junctions. In normal lymphoblast mitochondria, cristae are connected to the inner boundary membrane by slit-like openings. These slits had the same width as the cristae $(20-35 \mathrm{~nm})$ and a length of at least $50 \mathrm{~nm}$, but often exceeding $100 \mathrm{~nm}$ (Figure $6 a)$. The same features of cristae junctions were found in BTHS mitochondria (Figure 6b). Furthermore, the $150 \mathrm{~nm}$ sections showed that the large tubes of BTHS mitochondria were interconnected, enclosing a continuous cavity with varying diameter and multiple branches. The membrane surrounding this cavity originated from the cristae (Figure 6c).

\section{Discussion}

In this paper, we provide new insight into the ultrastructure of lymphoblast mitochondria from 


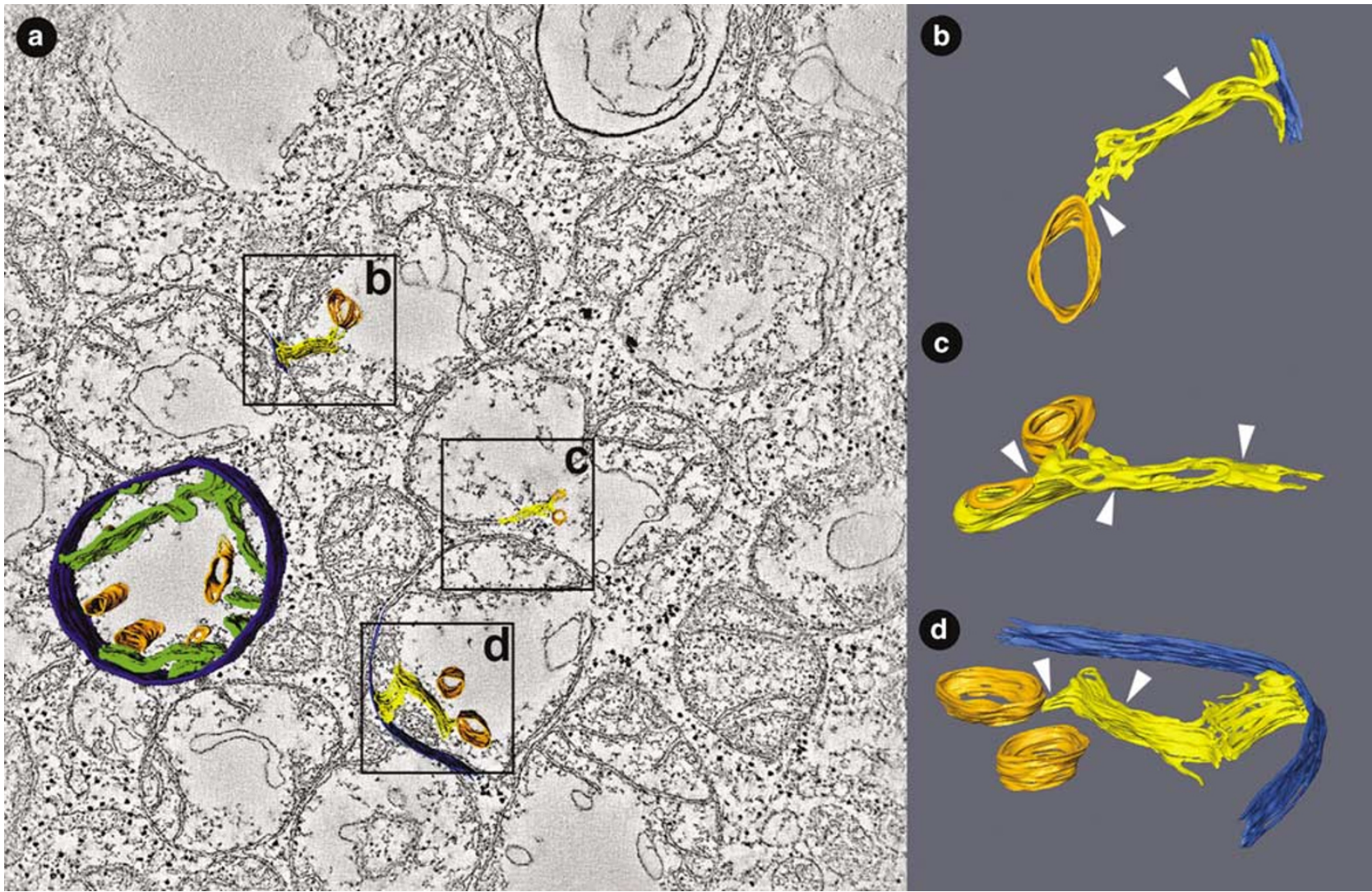

Figure 3 Tomogram of a cluster of fragmented mitochondria in a BTHS lymphoblast ( $50 \mathrm{~nm}$ section). (a) Slice of the tomogram with surface-rendered depiction of a 3-D model, showing the peripheral compartment (outer membrane + intermembrane space + inner boundary membrane) in dark blue, cristae in green, and large tubular structures in orange. The boxes $(0.4 \times 0.4 \mu \mathrm{m})$ mark three segmented details, in which the outer membrane is shown in bright blue, the inner membrane in yellow, and the tubular structures in orange. (b, c, d) 3-D models of membranes in the corresponding boxes. The images were magnified and rotated to reveal details of interest. White arrowheads point to zones of inner membrane adhesion. The large tubes (orange) are connected to collapsed cristae membranes.

both normal individuals and patients with BTHS. By using electron microscopic tomography, we were able to generate $3-\mathrm{D}$ views of portions of the intramitochondrial space. Although in our study, the reconstructed volumes were smaller than in previous tomography works, ${ }^{26-28}$ the 3 -D images enhanced the clarity of the structural framework and revealed novel pathologic features of BTHS mitochondria.

Pioneering electron microscopic tomography studies have demonstrated that mitochondrial cristae form either lamellar ${ }^{26-28}$ or amorphous ${ }^{23,29}$ compartments that connect to the mitochondrial periphery by cristae junctions. These junctions are often tubules that form circular or elliptical openings to the inner boundary membrane. ${ }^{24,26,27,29-33}$ In several mammalian mitochondria, these openings have a rather uniform size of $20-30 \mathrm{~nm} .{ }^{29-31}$ However, slotlike junctions with variable lateral extension have been observed in Neurospora mitochondria. ${ }^{28,34}$ Also, the cristae openings of liver mitochondria are not always circular, ${ }^{35,36}$ but may form elongated slits. ${ }^{37}$ Human lymphoblast mitochondria have primarily lamellar cristae with occasional tubular elements, and they have slot-like cristae junctions of variable dimensions (Figures 2,6). The morphology of lymphoblast mitochondria is consistent with the general concept that only segments of the cristae connect to the inner boundary membrane (cristae junctions). However, the dimensions of these cristae junctions may be tissue-specific or depend on the status of the mitochondria.

Most importantly, we found abundant evidence of abnormal cristae architecture in BTHS. This may be directly related to the deficiency of cardiolipin, because this lipid is concentrated in the inner mitochondrial membrane. The tomograms suggest a specific mechanism of cristae reorganization, namely obliteration of the intracristae space by adhesion of the two opposing cristae membranes, which compromises the continuity of the intracrista space and its connection to the peripheral intermembrane space. As the intracrista space is important for the transfer of substrates in and out of mitochondria, the collapsed cristae may be metabolically inactive. Cristae collapse may also be the explanation for the bundles of stacked inner membrane sheets, which were seen in heart mitochondria from BTHS patients ${ }^{19}$ and in wing muscles from tafazzin-deficient Drosophila. ${ }^{38}$ Although the mito- 

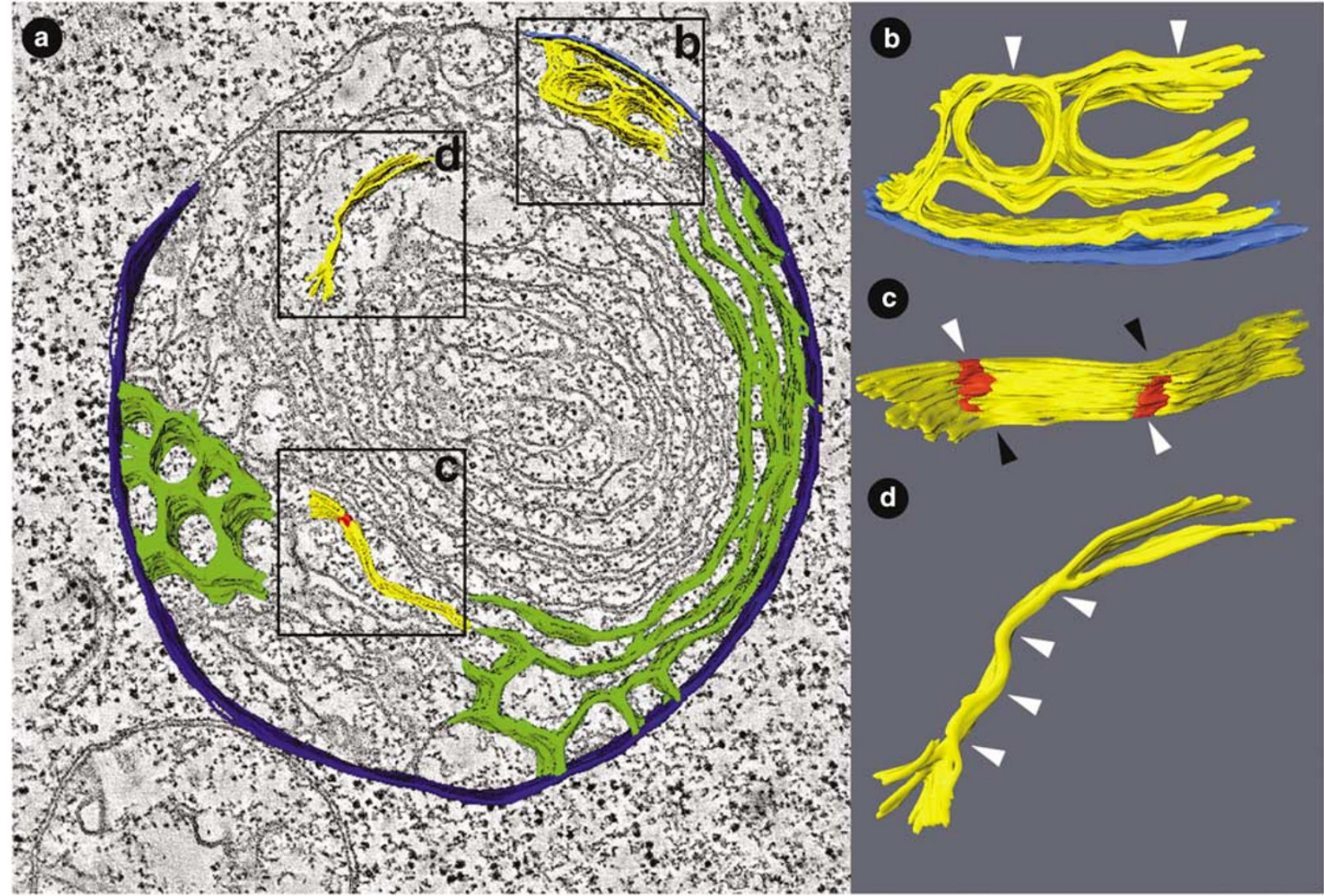

Figure 4 Tomogram of a giant mitochondrion in a BTHS lymphoblast ( $50 \mathrm{~nm}$ section). (a) Slice of the tomogram with surface-rendered depiction of a 3-D model, showing the peripheral compartment (outer membrane + intermembrane space + inner boundary membrane) in dark blue and the cristae in green. Two types of cristae arrangement are visible: a honeycomb pattern and a concentric pattern. The boxes $(0.4 \times 0.4 \mu \mathrm{m})$ mark three segmented details, in which the outer membrane is shown in bright blue and the inner membrane in yellow. (b, c, d) 3-D models of membranes in the corresponding boxes. The images were magnified and rotated to reveal details of interest. White arrowheads point to zones of inner membrane adhesion. In (c), the red zones mark adhesions of the inner membranes, white arrowheads point to areas with collapsed intracrista space, and black arrowheads point to areas with open intracrista space (see also Figure 5).

chondrial morphology in lymphoblasts is different from muscle and heart, the mechanism of cristae destruction may be similar, that is, obliteration of the intracristae space due to cristae membrane adhesion and stacking of the collapsed cristae. Perhaps, close apposition of membranes may be triggered by protein aggregation. In BTHS, deficiency of cardiolipin may predispose to protein aggregation because cardiolipin is essential for the correct supramolecular organization of protein complexes in the inner membrane. ${ }^{13,14}$ Alternatively, the absence of tafazzin itself may cause membrane adhesion because tafazzin is associated with inner and outer membrane surfaces of the intermembrane space. ${ }^{39}$

We do not know whether the ultrastructural changes of BTHS mitochondria are specific for this disease or whether they reflect a general pattern of destruction in pathologic mitochondria. Bissler et $a l^{19}$ reported that mitochondrial abnormalities of BTHS presented with a specific morphology that can be differentiated from other dilated cardiomyopathies. However, certain morphologic features seem to repeat themselves in many mitochondrial diseases, for instance, segregated compartments, similar to the large tubular structures in Figure $3 .^{21}$ Likewise, onion-shaped giant mitochondria have been observed in a variety of pathologic states, including ATP synthase deficiency, ${ }^{40}$ aging, hyperoxia, ${ }^{41}$ and others, although the exact morphology may not be identical in all instances. Onion-like structures likely reflect a lack of cristae junctions. ${ }^{42}$ BTHS mitochondria also show features that have previously been observed in association with apoptosis, ${ }^{36}$ such as matrix swelling and an apparent unfolding of the inner membrane (Figure 3) as well as an inversion of the cristae curvature (Figure 4).

In conclusion, we established that adhesions of the inner membranes are a dominant feature of pathologic mitochondria in BTHS lymphoblasts. Membrane adhesions occur in mitochondria of various sizes, including giant ones with onion-like appearance. More work is required to establish whether or not cristae collapse is a general phenomenon associated with mitochondrial diseases. 
46
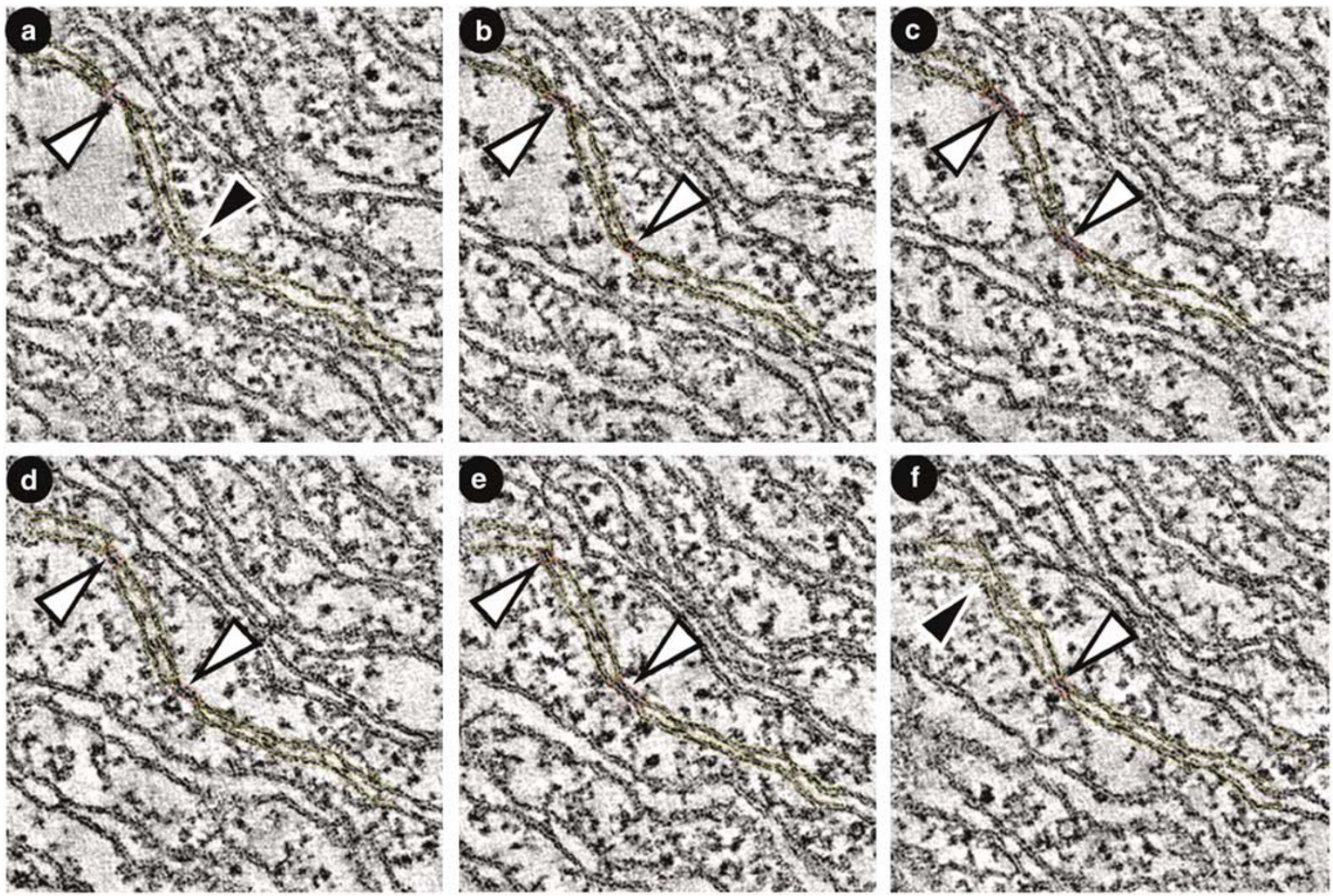

Figure 5 Representative slices through the tomogram showing segmentations used to create the model in Figure 4c. (a-f) The series of slices demonstrates the presence of adhesion zones between opposing inner membranes (white arrowheads). Adhesion zones alternate with zones that have an open intermembrane space (black arrowheads).

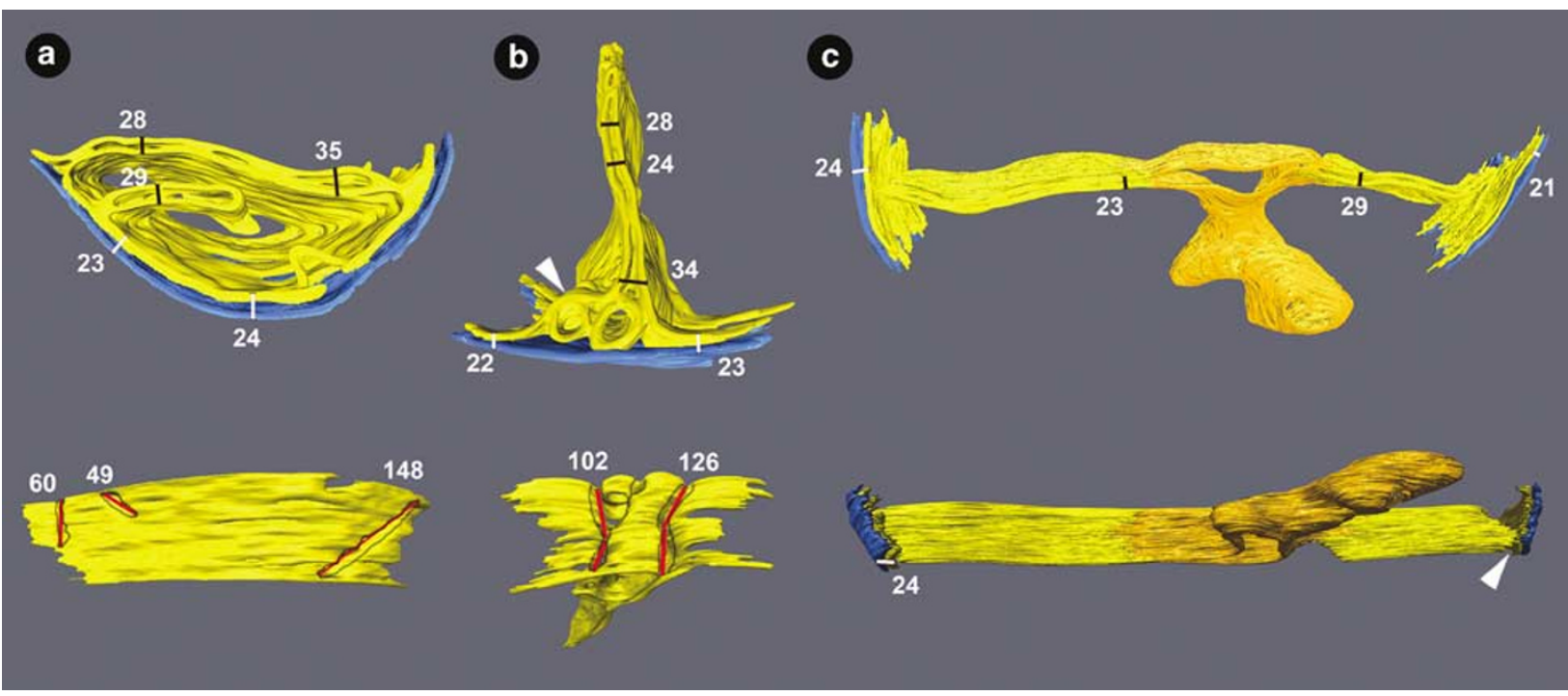

Figure 6 3-D models of tomograms obtained from $150 \mathrm{~nm}$ sections. The outer membranes are shown in blue and the inner membranes are shown in yellow. Each model is depicted in two views rotated approximately $90^{\circ}$ relative to each other. Numbers represent dimensions of the corresponding bars in $\mathrm{nm}$. White bars show the width of the mitochondrial periphery (outer membrane + intermembrane space + inner boundary membrane), black bars show the cristae width, and red bars show the length of cristae junctions at the level of the inner boundary membrane. (a) Mitochondrial membranes from a normal lymphoblast. In the lower image, the outer membrane was deleted to reveal the openings of three cristae junctions, outlined in gray. The left crista junction (60 nm) extends beyond the segmented volume. Dimensions in the upper image are consistent with previously published data. ${ }^{21,27,29,30}$ (b) Mitochondrial membranes from a BTHS lymphoblast. In the lower image, the outer membrane was deleted to reveal the openings of two cristae junctions, outlined in gray. The arrowhead points to a region of interconnected cristae with honeycomb pattern. (c) Mitochondrial membranes from a BTHS lymphoblast. Cristae are connected to a membrane (orange) that encloses a large cavity with multiple branches. This structure is specific to BTHS mitochondria and is also seen in Figure 3 (orange tubes). The arrowhead points to the lateral view of a crista junction. 


\section{Acknowledgements}

We are grateful to Dr Richard I Kelley (Kennedy Krieger Institute, Baltimore, MD, USA) for providing cultured lymphoblasts and for critically reading the manuscript. We are also grateful to Dr Carmen A Mannella (Wadsworth Center, Albany, NY, USA) for a critical discussion on our data. This work was supported by the National Institute of Health (1 R01 HL078788-01A1), the American Heart Association (grant-in-aid 0350126N) and the Barth Syndrome Foundation.

\section{References}

1 Barth PG, Scholte HR, Berden JA, et al. An X-linked mitochondrial disease affecting cardiac muscle, skeletal muscle and neutrophil leukocytes. J Neurol Sci 1983;62:327-355.

2 Kelley RI, Cheatham JP, Clark BJ, et al. X-linked dilated cardiomyopathy with neutropenia, growth retardation, and 3-methylglutaconic aciduria. J Pediatr 1991;119: 738-747.

3 Barth PG, Wanders RJA, Vreken P, et al. X-linked cardioskeletal myopathy and neutropenia (Barth syndrome) (MIM 302060). J Inher Metab Dis 1999; 22:555-567.

4 Bione S, D’Adamo P, Maestrini E, et al. A novel Xlinked gene, G4.5, is responsible for Barth syndrome. Nat Genet 1996;12:385-389.

$5 \mathrm{Ma} \mathrm{L}$, Vaz FM, Gu Z, et al. The human TAZ gene complements mitochondrial dysfunction in the yeast taz1 $\Delta$ mutant. Implications for Barth syndrome. J Biol Chem 2004;279:44394-44399.

6 Testet E, Laroche-Traineau J, Noubhani A, et al. Ypr140wp, the yeast tafazzin, displays a mitochondrial lysophosphatidylcholine (lyso-PC) acyltransferase activity related to triacylglycerol and mitochondrial lipid synthesis. Biochem J 2005;387:617-626.

7 Brandner K, Mick DU, Frazier AE, et al. Taz1, an outer mitochondrial membrane protein, affects stability and assembly of inner membrane protein complexesimplications for Barth syndrome. Mol Biol Cell 2005; 16:5202-5214.

8 Neuwald AF. Barth syndrome may be due to an acyltransferase deficiency. Curr Biol 1997;7:R465R466.

9 Vreken P, Valianpour F, Nijtmans LG, et al. Defective remodeling of cardiolipin and phosphatidylglycerol in Barth syndrome. Biochem Biophys Res Comm 2000;279: 378-382.

10 Schlame M, Towbin JA, Heerdt PM, et al. Deficiency of tetralinoleoyl-cardiolipin in Barth syndrome. Ann Neurol 2002;51:634-637.

11 Schlame M, Kelley RI, Feigenbaum A, et al. Phospholipid abnormalities in children with Barth syndrome. J Am Coll Cardiol 2003;42:1994-1999.

12 Schlame M, Rua D, Greenberg ML. The biosynthesis and functional role of cardiolipin. Progr Lipid Res 2000;39:257-288.

13 Zhang M, Mileykovskaya E, Dowhan W. Gluing the respiratory chain together. Cardiolipin is required for supercomplex formation in the inner mitochondrial membrane. J Biol Chem 2002;277:43553-43556.
14 Pfeiffer K, Gohil V, Stuart RA, et al. Cardiolipin stabilizes respiratory chain supercomplexes. J Biol Chem 2003;278:52873-52880.

15 Schlame M, Ren M, Xu Y, et al. Molecular symmetry in mitochondrial cardiolipins. Chem Phys Lipids 2005; 138:38-49.

16 Neustein HB, Lurie PR, Dahms B, et al. An X-linked recessive cardiomyopathy with abnormal mitochondria. Pediatrics 1979;64:24-29.

17 Hug G, Schwartz DC, Tuuri D. Sex-linked dilated cardiomyopathy, characteristic mitochondrial ultrastructure in liver, heart and muscle, effect of carnitine treatment. Pediatr Res 1990;27:20-28.

18 Christodoulou J, McInnes RR, Jay V, et al. Barth syndrome: clinical observation and genetic linkage studies. Am J Med Genet 1994;50:255-264.

19 Bissler JJ, Tsoras $\mathrm{M}$, Goring $\mathrm{HHH}$, et al. Infantile dilated X-linked cardiomyopathy, G4.5 mutations, altered lipids, and ultrastructural malformations of mitochondria in heart, liver, and skeletal muscle. Lab Invest 2002;82:335-344.

$20 \mathrm{Xu} \mathrm{Y,} \mathrm{Sutachan} \mathrm{JJ,} \mathrm{Plesken} \mathrm{H}$, et al. Characterization of lymphoblast mitochondria from patients with Barth syndrome. Lab Invest 2005;85:823-830.

21 Frey TG, Mannella CA. The internal structure of mitochondria. Trends Biochem Sci 2000;25:319-324.

22 Mannella CA. Introduction: our changing views of mitochondria. J Bioenerg Biomembr 2000;32:1-4.

23 Mannella CA, Pfeiffer DR, Bradshaw PC, et al. Topology of the mitochondrial inner membrane: dynamics and bioenergetic implications. IUBMB Life 2001;52:93-100.

24 Frey TG, Renken CW, Perkins GA. Insight into mitochondrial structure and functions from electron tomography. Biochim Biophys Acta 2002;1555: 196-203.

25 Kremer JR, Mastronarde DN, McIntosh JR. Computer visualization of three-dimensional image data using IMOD. J Struct Biol 1996;116:71-76.

26 Perkins GA, Renken CW, Martone M, et al. Electron tomography of neuronal mitochondria: 3D structure and organization of cristae and membrane contacts. J Struct Biol 1997;119:260-272.

27 Perkins GA, Song JY, Tarsa L, et al. Electron tomography of mitochondria from brown adipocytes reveals crista junctions. J Bioenerg Biomembr 1998;30: $431-442$.

28 Nicastro D, Frangakis AS, Typke D, et al. Cryo-electron tomography of Neurospora mitochondria. J Struct Biol 2000;129:48-56.

$29 \mathrm{He}$ L, Perkins GA, Poblenz AT, et al. Bcl-X $\mathrm{L}_{\mathrm{L}}$ overexpression blocks bax-mediated mitochondrial contact site formation and apoptosis in rod photoreceptors of led-exposed mice. Proc Natl Acad Sci USA 2003; 100:1022-1027.

30 Perkins GA, Renken CW, Frey TG, et al. Membrane architecture of mitochondria in neurons of the central nervous system. J Neurosci Res 2001;66:857-865.

31 Prince FP, Buttle KF. Mitochondrial structure in steroid-producing cells: three-dimensional reconstruction of human Leydig cell mitochondria by electron microscopic tomography. Anat Record 2004;2278A: 454-461.

32 Perkins GA, Ellisman MH, Fox DA. The structurefunction correlates of mammalian rod and cone photoreceptor mitochondria: observations and unanswered questions. Mitochondrion 2004;4:695-703. 
33 Perkins GA, Ellisman MH, Fox DA. Three-dimensional analysis of mouse rod and cone mitochondrial cristae architecture: bioenergetic and functional implications. Mol Vision 2003;9:60-73.

34 Perkins GA, Renken CW, van der Klei IJ, et al. Electron tomography of mitochondria after the arrest of protein import associated with Tom19 depletion. Eur J Cell Biol 2001;80:139-150.

35 Mootha VK, Wei MC, Buttle KF, et al. A reversible component of mitochondrial respiratory dysfunction in apoptosis can be rescued by exogenous cytochrome c. EMBO J 2001;20:661-671.

36 Scorrano L, Ashiya M, Buttle K, et al. A distinct pathway remodels mitochondrial cristae and mobilizes cytochrome $c$ during apoptosis. Dev Cell 2002; 2:55-67.

37 Hsieh C-E, Leith A, Mannella CA, et al. Towards highresolution three-dimensional imaging of native mam-

malian tissue: electron tomography of frozen-hydrated rat liver sections. J Struct Biol 2006;153:1-13.

$38 \mathrm{Xu} \mathrm{Y}$, Condell M, Plesken $\mathrm{H}$, et al. A Drosophila model of Barth syndrome. Proc Natl Acad Sci USA 2006;103:11584-11588.

39 Claypool SM, McCaffery JM, Koehler CM. Mitochondrial mislocalization and altered assembly of a cluster of Barth syndrome mutant tafazzins. J Cell Biol 2006;174:379-390.

40 Paumard P, Vaillier J, Coulary B, et al. The ATP synthase is involved in generating mitochondrial cristae morphology. EMBO J 2002;21:221-230.

41 Walker DW, Benzer S. Mitochondrial 'swirls' induced by oxygen stress and in the Drosophila mutant hyperswirl. Proc Natl Acad Sci USA 2004;101:10290-10295.

42 Mannella CA. Structure and dynamics of the mitochondrial inner membrane cristae. Biochim Biophys Acta 2006;1763:542-548. 